\title{
Relaciones virtuosas. Empresas y asociaciones frente a la Responsabilidad Social Corporativa
}

\author{
Laura Solito \\ Università degli Studi di Firenze
}

\section{Palabras clave}

Comunicación social; Responsabilidad Social Corporativa; Asociaciones voluntarias; Interacción; Relación comunicativa.

\section{Resumen}

La Responsabilidad Social Corporativa (RSC) emerge como un fenómeno comunicativo interesante, presentando ejemplos relevantes que muestran que es posible conciliar los valores éticos con los objetivos de las empresas. Si en el pasado este tipo de acciones se interpretaban como un intento de la empresa para conquistar al consumidor, sin que hubiera una implicación real en los temas sociales propuestos, a día de hoy se erige como objetivo la construcción de una relación diferente entre la empresa, el consumidor y el contexto social. En este trabajo se presentan los resultados de una investigación que estudia la comunicación de la RSC. Se han analizado en profundidad 10 proyectos realizados por 10 empresas y una decena de asociaciones. Para conseguir los objetivos de la investigación se ha utilizado una metodología de análisis cualitativo: a) el análisis de las páginas web de las asociaciones y de las empresas implicadas en los proyectos, para poder identificar las modalidades comunicativas de las iniciativas y enmarcar así el proyecto seleccionado en un contexto comunicativo más amplio para ambos socios; b) realización de entrevistas en profundidad de al menos un representante de cada uno de los socios (en total se han realizado 23 entrevistas); c) el estudio de los materiales utilizados en el proceso de producción y comunicación de la iniciativa y el análisis del contenido de los mismos. Los datos de los casos analizados y la articulación de los resultados obtenidos se han seleccionado, refutado e interpretado en base a un objetivo concreto: identificar, a pesar de su heterogeneidad ineludible, los elementos que comparten todos los proyectos y que favorecen la posibilidad de incrementar y reforzar el "espacio" que los proyectos de responsabilidad social corporativa están intentando conquistar en el escenario más amplio y variado de la comunicación social. Se considera que a la colaboración entre empresas y asociaciones es una relación comunicativa $y$, por tanto, se ha analizado desde el punto de vista relacional y dialógico. 


\title{
Virtuous relationship. Companies and associations in the corporate social responsibility
}

\section{Keywords}

Social communication; Corporate Social Responsibility; Volunterism Associations; Interaction; Relationship.

\begin{abstract}
Corporate social responsibility is coming out as an interesting communicative phenomenon, suggesting important examples as it's possible to bring out the aims of ethic values with business organization goals. If in the past this action was an attempt of the companies to win consumers without a real involvement of the social subjects suggested, nowadays it has to create a different relationship among companies, consumers and social context. This work shows the results of a research about the corporate social responsibility communication. Ten projects carried out by ten companies and by as many other associations have been analysed. The research used qualitative methods: a) the analysis of web sites of associations and companies involved in the projects, to identify and analyse the communicative strategies and arrange the selected project in the wider communicative context of both partners; b) interviews to a referent of each partner at least (23 interview have been carried out); c) the analysis of materials communication both partners. The wealth of the analysed experiences and the results achieved are here selected and interpreted as regards a precise aim: to identify -even if in their heterogeneity-those peculiarities you find in every project and that are able to increase and reinforce the "space» that projects of corporate social responsibility are hardly acquiring within the social communication. The partnership between business organizations and associations is considered a communicative relationship and so analysed from a relational and dialogic point of view.
\end{abstract}

\begin{abstract}
Autora
Laura Solito [laura.solito@unifi.it] es profesora titular de Sociología de los Procesos Culturales y Comunicativos en la Università degli Studi di Firenze, dónde, además, es directora del curso de Laurea Magistrales en Comunicación Estratégica.
\end{abstract}

Créditos: Artículo traducido del italiano por Sara Ortells Badenes. 
En este trabajo se presentan los resultados de una investigación que analiza la comunicación de Responsabilidad Social Corporativa (RSC). Se han analizado en profundidad 10 proyectos de responsabilidad social realizados por $10 \mathrm{em}$ presas y otras tantas asociaciones ${ }^{2}$. El corpus empírico ha sido seleccionado tomando como base dos criterios principales:

1. La representatividad con respecto a las modalidades principales de colaboración entre empresas y asociaciones sin ánimo de lucro. Se han primado aquellos casos en los que la empresa utiliza de manera más clara los bienes y servicios producidos para apoyar causas sociales específicas; los casos en los que la causa fomentada por el socio asociativo va unida claramente a los valores declarados de la empresa, es decir, aquellos en los que la empresa dona recursos, materiales o inmateriales, para apoyar iniciativas sin ánimo de lucro individuales y específicas (Cucco y otros, 2005).

2. La clara y evidente distinción de roles entre los dos socios en la gestión, realización y comunicación del propio proyecto ${ }^{3}$.

Para alcanzar los objetivos de la investigación se han utilizado herramientas de investigación cualitativa:

1. El análisis de las páginas web de las asociaciones y de las empresas implicadas en los proyectos, con la finalidad de identificar las modalidades comunicativas de las iniciativas para enmarcar el proyecto seleccionado en el contexto comunicativo más amplio posible para ambos socios. Además, el análisis de las páginas web ha ofrecido una serie de elementos útiles para esbozar la parrilla utilizada en las entrevistas.

2. Entrevistas en profundidad al menos a un representante de cada uno de los socios (en total se han realizado 23 entrevistas).

3. El estudio de los materiales de comunicación de la iniciativa y el análisis de su contenido.

La riqueza de los ejemplos analizados y la articulación de los resultados obtenidos se han seleccionado, refutado e interpretado en base a un objetivo concreto $^{4}$ : identificar, a pesar de su ineludible heterogeneidad, los elementos que contienen todos los proyectos y que están en disposición de incrementar y

2 Dada la imposibilidad de describir analíticamente cada una de las organizaciones implicadas en cada uno de los proyectos realizados, se indican los casos de estudio para la comprensión y contextualización de nuestras reflexiones: Andando a veglia, Fondazione Il Cuore si scioglie (Unicoop) y Associazione Nazionale delle Pubbliche Assistenze; A scuola di cuore, Acqua dell'Elba e Associazione Amico del Cuore; Biovit, Chimont Group e Legambiente; Carta Etica, Unicredit Group y Fondazione Banco Alimentare; Gift Twice, IKEA (Sesto fiorentino) y Associazione Toscana Tumori; 11 giro del mondo in 180 figurine, Coop y WWF; Immagina, Conad y Associazione Italiana Leucemie; La solidarietà vola alto, NeosAir e ADMO; Panda Club, Electrolux y WWF; Un gioiello per Tommasino, Nomination y Fondazione Tommasino Bacciotti.

3 Los criterios de selección que se han descrito están acompañados de un tercer factor preferencial del cliente, se trata del hecho de que al menos uno de los socios del proyecto sea de la región toscana, ya sea por procedencia, sede de trabajo o área geográfica en la que se desarrolla la iniciativa.

4 Para una descripción más completa del proyecto y de los resultados consultar: Solito, Materassi (2013). 
reforzar el «espacio» que los proyectos de RSC están intentando conquistar en el escenario más amplio y variado de la comunicación social.

\section{Con y sin ánimo de lucro: una unión en aumento}

En los últimos años, la cuestión de la Responsabilidad Social Corporativa (RSC) está emergiendo como un fenómeno comunicativo interesante, aunque todavía con ambigüedades y contradicciones, que propone ejemplos relevantes de cómo se pueden conciliar los valores éticos con los objetivos empresariales.

El fundamento de la actuación responsable se basa en la relación con el Otro. La responsabilidad corporativa no se fundamenta únicamente en la toma de decisiones racionales, las presiones y los incentivos por comportarse de una determinada manera, sino también en la capacidad de interactuar con los otros: «la relación entre la empresa y el entorno (social, político, natural) es tan profunda que la responsabilidad no es únicamente compatible con la lógica empresarial, sino que forma parte esencial de la programación de su estrategia» (Magatti, 1999: 14). La RSC debe ser entendida como «el deber de seguir aquellas políticas, de tomar aquellas decisiones, de seguir aquellas líneas de acción que se consideran deseables en función de los objetivos y los valores reconocidos por la sociedad» (Zattoni, Perrini, 2006: 536).

Si en el pasado está acción se interpretaba como un intento de la empresa de conquistar al cliente, sin tener una implicación real en los temas sociales propuestos, a día de hoy, también a raíz de los estudios de márquetin y de consumo, se ha convertido en una tendencia dividida, al menos en teoría, entre la construcción de una nueva relación entre los bienes producidos y los consumidores, la empresa y el entorno, y el mundo empresarial y el mundo social. Por tanto, la empresa, como sujeto integrado en el tejido social cuya acción resulta relevante, tiene en cuenta las características de los nuevos consumidores que aparecen, crecen y que, cada vez más, demuestran seleccionar las empresas y los productos también en base a sus consideraciones y valores éticos (Cevolini, 2001).

Y, por tanto, en esas mutaciones profundas que surgen entre los consumidores, en los mercados y, de manera más general, en la sociedad se insinúa y alimenta la atención hacía la comunicación de las responsabilidades sociales, y es en estas mismas mutaciones donde debe de encontrar apoyo y refuerzo, coherencia y legitimación.

De hecho, la atención de los consumidores hacia estos temas crece. Parece que con más frecuencia el consumo «actúa dotado de sentido», crea significados y prácticas sociales, critica y es consecuente, y ya no presta atención únicamente a la conveniencia y la satisfacción, sino que se muestra sensible con otros temas y nuevas dimensiones (Eurisko, 2003): la dimensión ética de los consumidores que, con un aumento exponencial, se va extendiendo a sectores cada vez más 
amplios de población (Douglas, 1996; Paltrinieri, 2004; Sassatelli, 2004; Di Nallo, Paltrinieri, 2006; Leonini, Sassatelli, 2008); los mercados, que dejan de ser simples lugares de transacción, y se convierten en lugares de conversación (Locke, Levine, Searls, Wienberger, 2001; Anderson, 2007); las empresas que enriquecen sus productos, bienes y servicios, con significados simbólicos y valores intangibles (Douglas, Isherwood, 1984; Floch, 1992; Lash, Urry, 1994). En definitiva, la ética y la responsabilidad social se convierten en dimensiones con las que competir, o se podría decir «competir nuevamente», desde una óptica profundamente diferente al significado que se le atribuye tradicionalmente y a la forma de actuar que ejercen habitualmente (Sacconi, Baldin, 2003).

En este escenario de transformaciones las aquí de manera abreviada llamadas empresas buscan concretar y hacer operativa la idea de «buena ciudadanía», entendida como la relación estrecha e indisoluble con el sistema social pero, sobre todo, también como el rol activo y contributivo a su propia creación, transformación, potenciación y enriquecimiento (Bruni, Zamagni, 2004; La Rosa, Morri, 2005). Un escenario, este último, sugestivo y estimulante pero que precisa, aunque no de manera urgente, una matización: no se trata de una empresa dispuesta a renunciar a su rol ni a su beneficio (Foglio, 2003; Porter, Krame, 2006): «la nueva y ventajosa sensibilidad y atención a lo social no puede, por tanto, ensombrecer el hecho de que la empresa se adentra en estos campos para conseguir mejorar sus objetivos... aquello que parece improbable es que la empresa y el márquetin, como ya hicieron en el pasado, puedan perseguir ahora intereses opuestos al respeto a lo social, alejándose de los significados sociales y de los símbolos del consumo» (Fabris, 2008: 225).

Desde esta perspectiva de unión entre empresa y social se inscriben todas aquellas iniciativas y proyectos, cada vez más numerosos, que, aunque tengan una naturaleza diferente y con diferentes niveles de implicación y responsabilidad, todavía están ampliando, enriqueciendo y diversificando el panorama de la RSC (Mattana, 2003; Molteni, Devigli, 2004; Perrini, 2006; Ferrari, Renna, Sobrero, 2009; Bonani, 2013).

En un contexto al que le falta madurar, pero que ya se ha extendido y presenta su versatilidad, aquellas iniciativas que involucran a más sujetos y que consiguen conciliar mundos diferentes (Quacquarelli, Paoletti, 2003), que, a pesar de mantener sus propias particularidades, encuentran en la comunicación social un punto de encuentro y de beneficio común, están adquiriendo especial relevancia y significado. Nos referimos especialmente a las interacciones entre el mundo de las asociaciones y el mundo de las empresas, que encuentran en el concepto clave de la «responsabilidad» una ocasión para reafirmar su propia identidad y construir un discurso público (Jonas, 1990). Una responsabilidad entendida como una práctica que precisa una respuesta, que se conecta con otras «realidades» con las que establece relaciones, una responsabilidad consciente de que dependiendo de cómo sea la reflexión continua sobre las propias acciones y la confrontación de las acciones de los otros se podrá consolidar el propio valor 
(Leccardi 2009). Estas afirmaciones van unidas a dos cuestiones fundamentales que contribuyen de alguna manera a explicar el desarrollo, el incremento y las transformaciones de este tipo de comunicación.

Sobre todo, la cuestión relativa a la construcción del discurso público y de la agenda de los problemas de relevancia colectiva.

La comunicación social ${ }^{5}$ tiene como objetivo dirigir la atención a los valores compartidos, a reafirmar su fuerza y centralidad, mostrándose como un instrumento potencialmente capaz de alimentar el discurso de la y en la esfera pública, además de contribuir a la construcción de la agenda de la atención y discusión pública (Marini, 2006; Bosco, 2012). De hecho, esta forma de comunicación, en el momento en el que centra la atención pública en objetos y temas sociales, creando, evidentemente, selecciones y definiciones «representa uno de los lugares en los que se debate, se elabora, se construye y define una "geografía" de los problemas de relevancia social» (Gadotti, 2005: 51). De hecho, con la pluralidad de voces que siempre la ha caracterizado, contribuye a construir un discurso público sobre las problemáticas de relevancia colectiva, y también de transformarlas en «problemas sociales» para incluirlos en la agenda de los actores políticos.

En este sentido parece claro que la comunicación de las responsabilidades sociales puede ser para la empresa una ocasión para reafirmar y fortalecer su propio papel de «emprendedor» de causas, de «enunciador legítimo de la definición de los problemas públicos»" de actor social que actúa, promueve y se «hace cargo».

En segundo lugar, hablar de comunicación de las responsabilidades sociales también implica hacer referencia a la relación difícil y controvertida entre comunicación y visibilidad, reconocimiento y legitimación. En esta esfera pública transformada y redefinida, surge con fuerza la exigencia por parte de todos los actores sociales de construir un rol público irrenunciable, una delimitación más clara de las propias obligaciones y funciones, además de la exigencia de confirmar el propio campo de intervención y la eficacia de las propias acciones, en resumen, la oportunidad de reforzar un proceso de identidad simbólica, construyendo, promoviendo y visibilizando la propia identidad y la propia imagen (Solito, 2010). Una esfera pública cada vez más densa, por tanto, «un lugar de exhibición pero en primera instancia de construcción, por parte de todos los que la habitan, de formas de reconocimiento basadas en el desarrollo y la visibilidad de la propia reputación» (Sorrentino, 2008: 69-70).

Por tanto, la comunicación deviene una fuente negociable a través de la que se define la relación con el contexto, se construye el reconocimiento y la visibilidad

5 Consultar: Cucco E., Pagani R. y Pasquali M. (2005), Primo rapporto sulla comunicazione sociale in Italia, Rai-Eri, Roma, y Cucco E., Pagani R., Pasquali M. y Soggia A. (2011), Secondo Rapporto sulla comunicazione sociale in Italia, Carocci, Roma. El primer y el segundo informe sobre comunicación social en Italia ofrecen elementos importantes de reflexión sobre estos temas, las problemáticas y las transformaciones de este ámbito cada vez más complejo y articulado de la comunicación.

6 Para confirmar que las problemáticas entre la intersección del papel de la empresa y lo social no es una novedad, consultar Semprini (1993). 
pública: que hace entendible la propia misión y acción social, las características que la distinguen, las motivaciones que la animan y la apoyan. En una palabra, para construir visibilidad pública, sin entender la visibilidad como un fin en sí mismo, sino buscada y construida para responder a una necesidad creciente de reconocimiento, utilizando diversificaciones, negociaciones y afirmaciones de la propia identidad: «hoy presentarse de manera eficaz frente a los otros cuyo consenso debe ser alimentado constantemente... ya no representa una opción posible, sino más bien un imperativo» (Thompson, 1998: 193).

La identidad ya no está limitada y circunscrita al «quién se es», sino que debe ser negociada y construida en relación con el contexto social y los propios interlocutores, para ampliar «espacios» de legitimación, «posicionarse», es decir, colocar la propia identidad, clara, distinta, única y creíble, y aquello que se lleva a cabo en la red de relaciones. Una identidad que se construye exponiéndose a los otros.

Y esto no es únicamente válido para las empresas, sino también para el mundo de las instituciones sin ánimo de lucro y el asociacionismo (Fazzi, 2000), que se ha transformado profundamente en los últimos años, y ha encontrado en esta dimensión comunicativa y relacional con el sector lucrativo una oportunidad para apoyar y dar nueva vida a su propia misión, y también para reforzar su progresiva confirmación como recurso importante e indiscutible para la sociedad civil. En un contexto cada vez más rico y plural de actores, de ámbitos de intervención, de causas sociales que promover y sensibilizar, la comunicación deviene cada vez más una exigencia y un recurso también para el asociacionismo $y$, en general, para una sociedad civil que «se caracteriza por su capacidad de no perder el contacto con la concreción de las relaciones y del cambio intersubjetivo» (Magatti, 2005: 84).

De hecho, parece haber una creciente sensibilidad hacia la comunicación incluso en el mundo no lucrativo (Francesconi, 2005), que se considera necesaria para conseguir los objetivos de las propias asociaciones (Salvini y Cordarz, 2005; Salvini y Corchia, 2012).

Se trata evidentemente de nuevas necesidades derivadas de las transformaciones de los roles del voluntariado, que se pueden sintetizar de manera eficaz a partir de la relevancia de las funciones cognitivas del contexto socio-cultural, que se expresa y realiza, sobre todo, mediante la capacidad de trabajar en red y de gestionar las dinámicas relacionales entre las asociaciones y, entre éstas y los otros sujetos sociales del territorio.

Una tarea que no es fácil, a casusa de múltiples factores como la competencia que caracteriza el mundo del voluntariado. Cada vez más ya se subraya que la comunicación se configura como una «oportunidad», una ventaja competitiva que puede ayudar a visibilizar las propias iniciativas, a difundir los valores que fundamentan la propia actividad, a construir notoriedad, legitimación y apoyo para el sujeto no lucrativo (Frisanco, Trassatti y Voterrani, 2000). 
La relación con el sector lucrativo puede convertirse, así, en un estímulo estratégico para convencer al público de la centralidad y la relevancia del propio campo de acción, para crear atención y sensibilidad, interés, conciencia, discusión, confrontación de ideas e identificación de posibles soluciones, para valorizar la propia presencia en el territorio. Pero, además, sobre todo, para conseguir los objetivos de reconocimiento y caracterización, identificación, legitimación y afirmación (Solito y Sorrentino, 2011). Objetivos que hoy en día, en un escenario cada vez más competitivo, únicamente son alcanzables enriqueciendo y caracterizando la propia identidad de los contenidos, proyectos, significados simbólicos y sentido. Y también en esta línea, si se entiende y se interpreta correctamente que el sector no lucrativo podrá conservar y, sobre todo, reforzar su protagonismo en la nueva sociedad.

A pesar de la crisis económica, parece que las buenas prácticas y las inversiones económicas en lo social por parte de las empresas crecen. La colaboración con el sector no lucrativo cada vez está más orientada y focalizada al intercambio de proyectos, de saberes y de oportunidades. De hecho, en los nuevos escenarios sociales, en los que se subrayan las transformaciones del sentido y de los significados del márquetin, en el que se abre camino el concepto de la ética corporativa y de su papel en el contexto en el que actúa, ya no es suficiente hacer regalos y donaciones sino unir estas acciones a la estrategia y a la cultura de empresa, inscribirlas en un proceso más amplio que implique a otros ámbitos en los que se defina la ética y la cultura de la responsabilidad corporativa. La cultura de la responsabilidad es una cosa que debe de ser compartida y que debe concernir a toda la empresa (Zamagni, 2004). Esto significa que no se realiza mediante acciones improvisadas y contingentes, sino que requiere visiones y gestiones estratégicas, de enmarcarse en un plano de programación coherente y compartido, en un proceso lógico, incluso en un proyecto: «...responsabilidad que se realiza más allá de la contribución y la libertad, incluso estrategias de atención dirigidas a los trabajadores, a las áreas del mundo de procedencia de las materias primas, a la manera de producir, a las condiciones de trabajo, a una retribución equitativa entre los trabajadores, a la eliminación de residuos y a la producción sostenible, etc. También precisa procesos de administración claros y transparentes y, sobre todo, de diálogo con los accionistas en cada una de las fases de las que se compone el camino hacia la responsabilidad» (Fabris, 2008: 495).

Un camino, por tanto, largo y complejo de entrada y salida hacia la autorreferencialidad pero que abre perspectivas y escenarios en los que no es difícil identificar fuertes motivaciones y estímulos entre las partes implicadas.

Como muestra la literatura y la experiencia, son múltiples los objetivos que se quieren y pueden conseguir mediante una gestión cuidada y estratégica de la unión entre una empresa y una institución sin ánimo de lucro. Si por parte de la empresa se trata de aquellos relacionados con las personas (motivaciones del personal, cohesiones, sentimiento de pertenencia, ambiente empresarial), con 
la comunidad (contribuir al desarrollo social/ambiental, apoyo, servicio), con la propia empresa (imagen, reputación de la identidad corporativa, relaciones y por último retorno económico), no menos relevante es el empuje que da a las asociaciones: visibilidad y legitimación, aumento de los recursos económicos y humanos, promoción de una causa social e implementación del propio rol en la sociedad.

Si por un lado la pluralidad y la heterogeneidad de las iniciativas que cada vez con más frecuencia implican a asociaciones y empresas evidencia, como se ha dicho, un contexto actual quizá más maduro y agitado, por el otro, florece la exigencia de valorar y comprender en profundidad la relación entre estos dos mundos: intentar comprender qué sucede «dentro» de esta relación, es decir, observar las premisas sobre las que se fundamenta, los modos y las formas mediante los que cobra vida, se estructura, perdura, evoluciona y se transforma.

\section{Los resultados: posibles convergencias}

Ya se ha subrayado de qué manera la atención a lo social y al binomio lucrativono lucrativo puede ser una nueva oportunidad no sólo para la empresa, sino también para el mundo no lucrativo: para reafirmar el propio rol y la propia acción, construir imagen, reputación, visibilidad, para proyectar y crear la propia identidad, los propios valores y las propias funciones. Y hacerse responsable.

De hecho, hacerse responsable, es una condición que fundamenta no solamente la visibilidad pública sino también la credibilidad, que cada vez más se busca y se consigue mediante el trabajo incesante de «construcción de las relaciones sociales» (Magatti, 2005).

Una transformación que impone el cambio de la lógica del oportunismo a la de la oportunidad. Oportunidad significa vivir la responsabilidad social como algo prioritario, no como un nuevo imperativo, un axioma, un comportamiento responsable o políticamente correcto por parte de la empresa, o como una manera de encontrar recursos para el asociacionismo, ni siquiera, evidentemente, como una estrategia de representación de sí misma, en la que determinadas señales y mensajes parecen útiles más por el hecho de «parecer» que por «mostrar» y «dar a conocer».

La comunicación de las responsabilidades sociales como oportunidad muestra la verdadera ruptura con el pasado, porque pone en el centro de sus objetivos la posibilidad de realizar un encuentro lucrativo, no únicamente instrumental, con la sociedad, sus necesidades, sus valores e incluso sus expectativas (Magatti y Monaci, 1999), un encuentro fruto de una «selección» libre y, como tal, impulsada por la intencionalidad, conocimiento e interés. 
De hecho, no basta con saber aprovechar esa oportunidad, ni elegir de manera intencionada colaborar y actuar con la comunidad: el éxito de una asociación entre empresa y mundo asociativo no es predecible.

El acuerdo entre los integrantes de la asociación y sus motivaciones recíprocas, son el resultado de un grupo heterogéneo de variables y factores que no únicamente dependen del contexto, sino también de las condiciones objetivas y estructurales dentro de las que el sujeto se mueve y actúa, y de las «disposiciones subjetivas», es decir, de la voluntad de perseguir de manera continua y coherente el objetivo de construir y gestionar las relaciones, de compartir.

A partir de estas consideraciones se fundamenta la perspectiva con la que hemos analizado la colaboración entre empresas y asociaciones: considerar la relación lucrativo-no lucrativo como una relación comunicativa y, por tanto, analizar esta relación desde un punto de vista relacional y dialógico.

De hecho, igual que cualquier relación comunicativa, la unión entre empresas y asociaciones se construye con el tiempo, a través de la conciencia y el conocimiento recíproco y la voluntad/capacidad de declinar objetivos diferentes en detrimento de fines comunes, la capacidad de relacionarse y referirse el uno al otro teniendo en cuenta las intenciones recíprocas, motivaciones, expectativas y diversidades (Gili y Colombo, 2012). Las relaciones comunicativas, además, prevén siempre un trabajo de gestión y respeto (Emirbayer, 1997; Donati, 1983, 1998, 2011; Crossley, 2011; Terenzi, 2012). El resultado de esta relación va más allá y es diferente a cada uno de los sujetos participantes: es el resultado de una acción recíproca.

Por tanto, relación, interacción y comunicación son los conceptos clave en torno a los que hemos desarrollado el análisis de la colaboración entre estos dos mundos, para identificar comportamientos, rutinas, y procedimientos prácticos llevados a cabo capaces de favorecer el «éxito» y la duración del proyecto.

\subsection{La colaboración como relación: dinámicas de construcción}

Ya se ha hecho referencia a la exigencia por parte de todos los sujetos sociales, dentro de las condiciones y características redefinidas del espacio público, de establecer relaciones con otros sujetos, así como también se han subrayado las transformaciones de los mercados, cada vez concebidos más como lugares de «conversación» y, por tanto, de dialogo y relaciones. Aquello que se pretende matizar es que las relaciones prevén un trabajo de construcción, de control y de respeto.

El contenido, las formas y las dinámicas de una relación pueden cambiar y ser también variables, pero como demuestra esta investigación la creación de una relación se fundamenta en una serie de presuposiciones y condiciones ineludibles. 


\section{- Reconocer al socio}

Desde el primer momento, y hasta el final, la relación es un camino de doble vía, es decir, se fundamenta en el conocimiento de los interlocutores, sobre la capacidad de escuchar las motivaciones, necesidades, expectativas y el diálogo entre pares, o sea, entre los socios. La construcción de las relaciones se refiere a la confianza, la reputación, la fiabilidad de los sujetos como fuente fundamental de estimulación.

El conocimiento es seguramente el primer paso hacia la colaboración: se tiene que saber a qué se dedica el socio potencial, si se recuerda una actividad específica, si se relaciona con un contexto determinado, si se asocia a un nombre, a una cara, a un logo, etc... Cada vez con más frecuencia para las empresas y, cada vez más, para las asociaciones, podremos hablar de «relevancia de marca», que debe de entenderse como marca o logo, es decir, de manera general debe de interpretarse como el «currículo» de la organización. Esta consideración implica tener en cuenta todos aquellos factores que caracterizan el trabajo de comunicación de las organizaciones antes de «abrirse» a la comunicación de las responsabilidades sociales y que contribuyen a la creación de la identidad, la visibilidad, la reputación, el reconocimiento y la credibilidad. Ya sea desde el punto de vista empresarial o asociativo, se advierte la exigencia de entender si el otro sujeto puede considerarse eventualmente como un socio creíble. Creíble en el sentido de que sea fiable. Utilizando diferentes niveles de formalizaciones, más extenso en los casos de las grandes empresas o fundaciones y más simples en el caso de las empresas o asociaciones más pequeñas o nuevas en el sector, todas las personas entrevistadas coinciden en su respuesta, en muy pocos casos se han sumado a una propuesta o han promovido alguna iniciativa o proyecto, sin haber investigado o valorado previamente la reputación de los posibles socios del proyecto.

\section{- Reconocer y valorar la diversidad}

La relación se desarrolla y se sostiene a partir de la capacidad de reconocer y mantener las distintas identidades de los interlocutores, en términos de valores, objetivos, diversidades organizativas, competencias y recursos. Se trata de un punto muy importante, de hecho a menudo es motivo de malentendidos y errores, miedos e estancamiento.

Cada uno tiene su propia identidad, historia, misión y visión. El asociacionismo no es un proceso de identificación o de fusión con el otro, sino de reconocimiento del otro, sin que implique la renuncia a la propia especificidad, ni la «contaminación» de los propios principios. El «valor añadido» de una asociación es precisamente la contaminación entre diferencias y la confrontación entre realidades diversas no únicamente en términos de valores y misión sino también de hábitos y procesos operativos, de objetivos de productividad. Y el contraste 
con una empresa organizacional muy diversa de sí misma puede favorecer la capacidad reflexiva, el conocimiento y valorización de las propias especificaciones y potencialidades, y la revisión de los modelos organizativos.

- Concreción de las acciones

No se trata de una relación abstracta, no se basa en principios y buenos propósitos sino que debe de ser capaz de «traducirse» en acciones visibles y concretas. Se trata de un requisito que procede normalmente del mundo empresarial. De hecho, muchos de los entrevistados desaconsejan pedir ayuda de manera genérica para apoyar una asociación o causa; por el contrario, es importante darle prioridad a un proyecto, a una acción, a propuestas concretas, «visibles» en los resultados que podrían conseguirse y con objetivos cuantificables. Por tanto, no ha lugar a la imprecisión ni a la generalidad.

Las relaciones y, por tanto, el asociacionismo, no pueden ser improvisadas, porque la unión entre mundos tan dispares precisa de acciones no banales desde el punto de vista de la gestión. Se precisa tener una previsión a largo plazo y preparación, y como se explicará más adelante capacidad para «apoyarlas».

\subsection{La asociación como interacción: la realización de un proyecto}

Una relación es la «acción entre» dos o más sujetos. Por tanto, reciprocidad, intercambio, transparencia, disponibilidad para colaborar e implicación de todos aunque, evidentemente, de manera diferenciada. Es necesario pasar de una lógica asimétrica a una lógica simétrica, de reciprocidad y colaboración, que debe de guiar la relación. Se trata del resultado de una acción recíproca.

De hecho, la construcción de una relación se configura como un acto voluntario (pero no unilateral) que precisa capacidad de gestión, de continuar con la acción, y de reconocer el valor añadido.

El reparto de las acciones y de los objetivos de comunicación y la colaboración entre los socios se definen como elementos indispensables e imprescindibles para asegurar el éxito de un proyecto de responsabilidad social. Resulta evidente que enfatizar estas condiciones no significa que todo deba de hacerse conjuntamente, al contrario, el sentido y el significado de la colaboración se sustenta por la capacidad de identificar las experiencias, competencias y habilidades, atribuir funciones y responsabilidades aprovechando y potenciando la especificidad, creando sinergias.

Se trata de un momento delicado y complejo: se reconocen las características y particularidades, se marcan los límites, se negocia las diferencias y las exigencias. Más allá de la consideraciones obvias de que la modalidad de gestión de las acciones previstas en el proyecto y de las relaciones entre los dos socios 
puedan mutar en función de la variaciones estructurales de las dimensiones de la empresa o la asociación, la tipología del proyecto, la cantidad de iniciativas y acciones de comunicación, lo que realmente interesa subrayar son las modalidades de relación entre los dos socios.

Aunque la relación entre los socios en la gestión de los proyectos parece seguir caminos diferenciados, a partir de la investigación realizada se ha detectado que el asociacionismo para alcanzar el éxito deseado no precisa una división paritaria, en término de carga de trabajo, de las funciones o trabajar constantemente de manera conjunta. El respeto mutuo y el reconocimiento del valor que tiene el otro socio: son los aspectos que deben de tenerse en cuenta y, por tanto, las modalidades operativas que realmente consiguen resaltar la productividad de cada uno. La reciprocidad en una actividad de comunicación social es posible si ambos promotores reconocen el papel de coprotagonista del otro, en otras palabras, cuando la relación se construye sobre el terreno del respeto y de la apreciación recíproca.

Éstas emergen como las condiciones ideales para la evolución hacia una relación madura, no únicamente porque los sujetos se reconozcan recíprocamente como socios sino, sobre todo, porque se tiende a:

- La construcción de un modelo organizativo dirigido a la gestión del proyecto y de su comunicación.

- La integración de los objetivos y de las herramientas de comunicación.

- La flexibilidad y la producción de intercambio.

La gestión de las relaciones como elemento base de la realización del proyecto precisa prestar atención a algunas variables importantes surgidas como resultado del análisis de los proyectos:

- La coordinación, se trata de un momento importante de conexión que facilita y monitoriza las fases de realización, los tiempos y tipos de encuentro entre los socios. De hecho, dependiendo del tipo de asociacionismo y, sobre todo, en aquellos más complejos, se ha visto que puede resultar determinante para alcanzar los objetivos fijados la presencia de una figura de conexión que «supervise» el proyecto.

- La implicación interna. Abrirse a la comunicación, emprender un camino hacia la construcción de una relación con el contexto y los interlocutores externos a la organización tiene como consecuencia inevitable la necesidad de hacer frente a dinámicas internas de relaciones y de trabajo entre las diferentes partes de la organización. La comunicación externa, con la finalidad de ofrecer información sobre la organización y también de construir la propia imagen y reputación y la posibilidad de basar las relaciones sobre la credibilidad y confianza «impone» de algún modo prestar atención al «interior» de la organización: el conocimiento y la implicación por parte de los 
trabajadores son estrategias estimulantes para conseguir los objetivos, la inversión en capital humano, de hecho, puede reducir los conflictos internos y tener consecuencias positivas en la productividad.

En el aspecto empresarial las mismas iniciativas y proyectos de responsabilidad social parecen reforzar la relación con los trabajadores: su implicación en la causa social aumenta la motivación, favorece la fidelización del proyecto y permite alcanzar objetivos más amplios.

De hecho, los proyectos analizados confirman la importancia de la implicación interna como una condición prioritaria para apoyar y reforzar el proyecto, para generar motivación y atención sobre la causa. Es más, se convierte en una herramienta última de transparencia, no únicamente por los motivos que acabamos de señalar sino también por la «funcionalidad» de la realización misma del proyecto. En algunos casos, de hecho, los proyectos prevén la participación activa de los trabajadores precisando por tanto una información minuciosa en el seno de la empresa y de la asociación.

La implicación puede contemplar también iniciativas más o menos formalizadas enfocadas a convertir a los trabajadores en actores protagonistas del propio proyecto, como por ejemplo los cursos de formación. O, la adhesión espontánea del personal (sobre todo en el caso de las empresas) al proyecto y a su modalidad de actuación que se revela como un instrumento capaz de crear una "proximidad simbólica» a la causa propuesta.

Por el contrario, sobre todo en algunas asociaciones de voluntariado, se ha identificado la falta de implicación interna y de la participación como un signo de crítica y debilidad.

- Superar las dificultades. Si bien la elección del socio se basa fundamentalmente en la coincidencia de los valores, también se da el caso de que en la fase producción pueden entrar en conflicto los puntos de vista de emprendedores y asociativos. Más que hablar de conflictos en sí mismo sería mejor hacerlo de «confrontaciones necesarias», de momentos de diálogo en los que los socios «negocian» no tanto los valores en los que se basa la relación, que de otro modo dejaría de existir, sino sobre los contenidos del proyecto, la modalidad de acción, la elección comunicativa. Se trata de un momento muy importante en el que los dos sujetos pertenecientes a dos universos culturales diferentes, con objetivos, finalidades, interlocutores y organizaciones diferentes, se ponen en contacto y empiezan a conocerse.

Los problemas que dificultan las relaciones consisten, según las empresas, en la autorreferencialidad, la rigidez y la incapacidad por parte de las asociaciones de conciliar su punto de vista con otros posibles. En cambio, las asociaciones ven la naturaleza lucrativa como el «pecado original», que en vez de complementar, se contrapone a los objetivos y al papel de las organizaciones filantrópicas, o a los objetivos no lucrativos. Esta es la diferencia cultural que se subraya de 
manera más frecuente que dificulta la creación de proyectos comunes y, sobre todo, impide reunir aquellas partes y especificidades que desde una óptica de complementación pueden favorecer una acción compartida sobre un tema, una causa o un territorio común.

La gestión de la actividad de comunicación del proyecto deviene un momento delicado y complejo en el que pueden surgir dificultades y críticas, puntos de vista, aproximaciones, mentalidades y, sobre todo, objetivos comunicativos legítimamente diferentes entre los socios. Sin dar pie a conflictos que invaliden el proyecto y su continuación estos momentos se configuran, por el contrario, como ocasiones importantes para reconocer la diversidad, reforzar el propio interés por el proyecto y su producción, para negociar puntos de vista y elecciones comunicativas diferentes, aunque sin renunciar a la propia especificidad y a su propio rol.

En todos los proyectos analizados no se hace nunca referencia a las dificultades relacionadas con problemas económicos y/o práctico-operativos, al contrario, allí donde se manifiestan, las críticas están relacionadas básicamente en dos ámbitos:

- Intereses comunicativos divergentes

- Problemas de lenguaje

Los primeros se originan a partir de las diferencias de identidad que caracterizan ambos mundos. La crítica más mencionada parece ser el riesgo de incongruencia entre la misión de la asociación y una aproximación excesivamente empresarial orientada por la empresa, como tal, considerada compatible con los temas sociales.

No faltan las ocasiones en las que es la empresa la que encuentra dificultades para conciliar las profesionalidades internas, las exigencias de las empresas de comunicación y márquetin con la inevitable mayor flexibilidad requerida por un socio, el de la asociación, necesariamente más atento a la disponibilidad de recursos e, incluso en ocasiones, caracterizado por competencias de dirección y comunicación menos estructuradas y formales, o incluso inexistentes.

En ocasiones, las asociaciones destacan las diferencias de lenguaje. Para el resto, los resultados confirman una sensibilidad creciente del asociacionismo de voluntariado hacia la modalidad y los lenguajes mediante los que comunicar la propia misión, las elecciones efectuadas y los objetivos conseguidos, la propia voluntad y la acción cotidiana.

\subsection{La comunicación: una dimensión constituyente y transversal}

Dado todo lo que se ha explicado, incluso un lector distraído puede entender como la comunicación, entendida prioritariamente como la capacidad de cons- 
truir y gestionar «relaciones», es una parte constituyente, una dimensión esencial e intrínseca de una relación de responsabilidad social.

De esta manera, la idea de la comunicación como herramienta parece lejana, más o menos potente, para reconstruir de manera rápida la fachada y la imagen, se trata de una cuestión de solidez e identidad, «acción» del y en el propio proyecto, hasta el punto que resulta difícil distinguir las actividades de comunicación con las acciones operativas que la caracterizan.

La gestión de un proyecto de responsabilidad social precisa, por tanto, una constante focalización de la atención hacia la comunicación.

La relación entre la empresa e institución sin ánimo de lucro, lo que resulta, como suele sostenerse, es una tendencia que se desarrolla por un camino que cumple las siguientes características: estratégico, elegido de manera voluntaria y consciente, estructurado, estructurado en fases y objetivos específicos, coherente, apostando por la sintonía y la sinergia entre las acciones y entre las misiones empresariales y asociativas, identificable, que se pueda presentar y valorar, compartido, precisa la colaboración y la implicación de todo, y en resumen, pero no por ello menos importante, comunicable, el proyecto se tiene que poder incluir en el contexto ambiental en el que se actúa y del que debe nutrir sus respuestas (Fabris, 2008).

En resumen, si por un lado, como se ha explicado, la comunicación y la calidad de las relaciones que se instauran determinan los contenidos y el desarrollo del propio proyecto, por otro, el proyecto debe de poder ser comunicado al público, a los destinatarios finales, y de manera general a los accionistas.

Tener un sistema de comunicación eficaz se configura como un factor fundamental para el éxito, naturalmente con la condición de que no sea discontinúo y subordinado a otros intereses y objetivos, y que esté fuertemente integrado en el proceso de actuación y la planificación del proyecto. De esta manera, la comunicación entra en una lógica de proceso:

- Que se inicia antes que la propia asociación y que el proyecto. Las actividades de comunicación autoproducida por los sujetos para reafirmar la propia identidad, obtener reconocimiento, dar a conocer el trabajo constituyen elementos, junto con otros evidentemente, sobre los que normalmente se pueden basar los criterios de selección del socio: la notoriedad de la «marca» y la credibilidad.

- Se desarrolla durante la realización de la asociación, gestionando las relaciones e implicando a las organizaciones.

- Se consolida dando a conocer e informando al público objetivo.

La comunicación, por tanto, es un sistema que precisa la integración y la planificación: la gestión de las relaciones (entre los socios y entre éstos y los accionistas) y los instrumentos de comunicación activados para dar a conocer, informar 
y promover son parte esencial del proyecto, porque integran estrategias y acciones sobre la base de los objetivos conocidos y compartidos.

Parece lógico que, aunque esté seleccionado e ideado conscientemente, gestionado y administrado con voluntad y coherencia por parte de los dos socios, el éxito del proyecto de una iniciativa no está garantizado. Estar atento, la previsión a largo plazo, identificar los objetivos, las herramientas de información y el público objetivo pueden ayudar a orientar la acción comunicativa para promover y publicitar, atraer la atención y la curiosidad, y explicar de un modo coherente y creíble la iniciativa. Desde este punto de vista la elaboración de una herramienta informativas ad hoc, las relaciones con los medios de comunicación, recurrir a las recomendaciones pueden constituir momentos delicados e incluso estratégicos.

Adoptar una modalidad de comunicación que utilice e integre un pluralidad de herramientas, capaces de dirigirse a públicos diversos, es una condición imprescindible para cualquier iniciativa o actividad comunicativa.

Hay tantas herramientas disponibles, algunas incluso innovadoras. Los proyectos que hemos analizado son conscientes de esta riqueza y variedad: de hecho, el abanico de herramientas citado por los entrevistados es tan amplio que podemos sintetizarlas en estas macrocategorías:

- Uso de las herramientas de comunicación interna y externa previamente presentes en las empresas: revistas, newsletters, revistas internas: se trata de una amplia disponibilidad para utilizar herramientas propias de comunicación por parte de las empresas y de las asociaciones que estratégicamente pueden resultar exitosas en relación a los objetivos de visibilidad del proyecto, ya sea para reforzar y compartir la sociedad, o para racionalizar y contener los gastos.

- Herramientas creadas ad hoc para el proyecto: panfletos, trípticos, folletos, posters y pancartas publicitarias. Herramientas en las que frecuentemente hay espacio para la creatividad o la experimentación con nuevos lenguajes, aunque también son herramientas con las que es más fácil hacer visible el logo y todos los símbolos que contribuyen a reforzar y hacer reconocible la identidad del proyecto.

- Eventos, seminarios específicos, gadgets: también están presentes en los proyectos analizados, cada vez más utilizados en el mundo de las asociaciones, que con esfuerzos organizativos de muchos de los contenidos favorecen la visibilidad, la notoriedad y la participación: organizaciones de encuentros o fiestas organizadas para presentar y dar a conocer el proyecto pero, sobre todo, para reafirmar la solidez de la asociación, seminarios especializados y de profundización de temas relacionados con el proyecto dirigidos a un target específico; gadgets para recordar, sensibilizar, y también para involucrar y motivar al personal interno.

- Los media. 
En lo que concierne a los media emergen dos consideraciones importantes. La primera está relacionada en general con el uso prevalente de la web. Junto con la relevancia del sitio web para promover los proyectos de responsabilidad social se abre camino también el uso de las redes sociales. En los proyectos analizados se ve claramente la concienciación del papel de la web social, su capacidad para ampliar el panorama de las elecciones posibles para llegar a segmentos de población específicos, para atraer y mantener viva la atención, a costes limitados, con lenguajes precisos y diversificados. El uso de la web también se refuerza con la percepción del poco espacio que tienen los medios tradicionales (prensa y televisión, especialmente) para dedicar a las iniciativas de responsabilidad social.

De esta manera surge el importante y constante trabajo de los gabinetes de prensa y las relaciones con los medios. Un trabajo que, como confirman nuestras entrevistas, parece encontrar respuesta en los medios locales, muchas veces gracias a las relaciones personales, aunque también a la prensa especializada en el sector.

\section{- La recomendación}

Tampoco falta en nuestros proyectos, aunque en pocos casos, estrategias comunicativas que apuntan a la implicación de expertos en la actividad de la información y la divulgación, investigadores de prestigio y autorrelevancia en el ámbito científico, actores y/o personajes famosos, o incluso políticos locales. La recomendación aparece como una elección estratégica, un valor añadido para reforzar la credibilidad y la seriedad.

A partir de todo lo que se ha explicado, surge con claridad el papel de la comunicación como una dimensión intrínseca y transversal de los proyectos de responsabilidad social: contribuye al lanzamiento y a la calidad de las relaciones, a los contenidos, al desarrollo y finalmente al éxito del proyecto. Es parte integrante del proceso de proyección, realización, valoración, visibilidad y promoción de un proyecto de responsabilidad social.

La aproximación relacional y dialógica al estudio de la responsabilidad social que se ha propuesto ha ayudado a focalizar la complejidad, en cuanto a proceso articulado y multiforme, que implica la identidad, la especificidad organizativa, los conocimientos, las competencias y las habilidades entre ellas aun siendo profundamente diferentes.

Más que conclusiones, por tanto, las reflexiones que se explican aquí seguramente necesitan nuevas y estimulantes hipótesis de investigación: para aumentar, mejorar y reforzar aquel «espacio» que los proyectos de responsabilidad social están intentando conquistar en el escenario más amplio y variado de la comunicación social. Un espacio que dado que es plural, diversificado y articulado necesita que se le preste atención, orientación y capacidad para identificar las señales y rutas que debe de recorrer para dar vigor y energía renovada a 
una relación entre dos mundos, el empresarial y el de la asociación, hasta ayer diferentes y muy distantes... hoy, diferentes pero un poco más cercanos.

\section{Referencias bibliográficas}

AA.VV. (2012). Tra $i$ due mondi. Storia, evoluzione e prospettive del rapporto tra profit e non profit in Italia. Milano: SDA Bocconi, Rapporto 2012.

Anderson, C. (2007). La coda lunga. Milano: Codice.

Balmer J.M.; Gray E.R. (1999). Corporate Identity And Corporate Communications: Creating a Comparative Advantage. En: Corporate Communication: An International Journal, $\mathrm{n}^{0}$ 4, 171-176.

Bernocchi, R. (2001). La comunicazione delle organizzazioni non profit. En: Gadotti, G. La comunicazione sociale. Soggetti, strumenti e linguaggi. Milano: Arcipelago ed.

Bonani, G.P. (2013). L’impresa aumentata. Caos e responsabilità della comunicazione d’impresa nell'età social. Milano: F. Angeli.

Bosco, N. (2012). Non si discute. Forme e strategie dei discorsi pubblici. Torino: Rosenberg \& Sellier.

Bruni, L.; Zamagni, S. (2004). Economia civile. Efficienza, equità, felicità pubblica. Bologna: Il Mulino.

Cevolini A. (2001). Politiche sociali, rischio, responsabilità: la responsabilità sociale nelle organizzazioni della società civile. En: Studi di sociologia, $\mathrm{n}^{\mathrm{0}} 4$, 465-478.

Corchia, L. (a cura di) (2012). I bisogni delle organizzazioni di volontariato. Firenze: Cesvot.

Crossley, N. (2011). Towards Relational Sociology. London and New York: Routledge.

Cucco, E. ; Pagani R. y Pasquali M. (2005). Primo rapporto sulla comunicazione sociale in Italia. Roma: Rai-Eri.

Cucc, E.; Pagani, R.; Pasquali, M. y Soggia, A. (2011). Secondo Rapporto sulla comunicazione sociale in Italia. Roma: Carocci.

Di Nallo, E.; Paltrinieri, R. (a cura di) (2006). Cum sumo. Prospettive di analisi del consumo nella società globale. Milano: Franco Angeli.

Donati, P. (1998).Teoria relazionale della società, Milano: Franco Angeli.

Douglas, M. (1996). Questioni di gusto. Bologna: Il Mulino. 
Douglas, M.; Isherwood, B. (1984). Il mondo delle cose. Oggetti, valori, consumo. Bologna: Il Mulino.

Emirbayer, M. (1997). Manifesto for a relational sociology. En: American Journal of Sociology, $\mathrm{n}^{\circ} 2,281-317$.

Eurisko (2003). I consumatori italiani e la responsabilità sociale delle imprese. En: Milano: CSR Monitor.

Fabris, A. (2006).Etica della comunicazione. Roma: Carocci.

Fabris, G. (2008). Societing, il marketing nella società postmoderna, Milano: Egea.

Faccioli, F.; D’Ambrosi, L. y Massoli, L. (2007). Voci della ribalta. Comunicazione sociale, processi inclusivi e partecipazione. Roma: Ed. Scientifiche italiane.

Fazzi, L. (a cura di) (2000). Cultura organizzativa del nonprofit, Milano: Franco Angeli.

Ferrari, L.; Renna, S. y Sobrero R. (2009). Oltre la CSR. Milano: ISEDI.

Floch, J.M., (1992). Semiotica, marketing e comunicazione. Dietro i segni, le strategie, Milano: Franco Angeli.

Foglio A. (2003). Il marketing non profit: strategie e politiche di marketing per associazioni ed imprese non profit. Milano: Franco Angeli.

Francesconi, A. (2007).Comunicare il valore dell'azienda non profit. Padova: CEDAM.

Frisanco, R.; Trasatti, S.; Volterrani,A. (a cura di (2000). La voce del volontariato. Indagine nazionale su organizzazioni di volontariato e comunicazione. Roma: Fondazione Italiana per il Volontariato.

Gadotti G. (a cura di) (2005). La comunicazione sociale. Soggetti, strumenti, linguaggi, Milano: Arcipelago,

Gili, G.; Colombo, F. (2012). Cultura, comunicazione e società. Brescia: La Scuola.

Kotler, P.; Andreasen, A. (1998). Marketing per le organizzazioni non profit. Milano: Il Sole 24 Ore.

Lalli, P. (2008). Imparziali ma non indifferenti, Faenza: HomelessBook.

La Rosa, M.; Morri, L. (2005). Etica economica e sociale. Letture e documenti. Milano: Franco Angeli.

Lash, S.; Urry, J. (1994). Economies of Signs and Space, London: Sage.

Leccardi, C. (2000). La responsabilità riflessiva. En: Melucci A. (a cura di). Parole chiave. Per un nuovo lessico delle scienze sociali, Roma: Carocci. 
Leonini, L.; Sassatelli R. (a cura di) (2008). Il consumo critico. Significati, pratiche, reti. Roma-Bari: Laterza.

Locke, C.; Levine, R.; Searls, D. y Wienberger, D. (2001). The Cluetrain Manifesto. The End of Business as Usual. New York: Peseus Books Group.

Magatti, M. (2005). Il potere istituente della società civile. Roma: Laterza.

Magatti, M. (1999). L’impresa della responsabilità. Linee per la ricerca. Invito ad assolvere un vizio. En: Magatti, M.; Monaco, M. (a cura di). L’impresa responsabile, Milano: Bollati Boringhieri.

Marini, R. (2006). Mass media e discussione pubblica. Le teorie dell'agenda setting. Roma-Bari: Laterza.

Mattana G. (2003). La Responsabilità sociale d'impresa: un forte crescendo di interesse, una grande sfida, anche per la qualità. En: Qualità, $n^{0} 4$.

Molteni, M.; Devigli D. (2004). Il Cause Related Marketing nella strategia d'impresa. Milano: Franco Angeli,

Morcellini, M.; Mazza B. (a cura di) (2008). Oltre l'individualismo. Comunicazione, nuovi diritti e capitale sociale. Milano: Franco Angeli.

Paltrinieri, R. (2004). Consumo e globalizzazione. Roma: Carocci.

Perrini, F. (2006). Responsabilità sociale d’impresa. Milano: Egea.

Porter, M.E.; Kramer, M.R. (2006). Strategy and society: the link between competitive advantage and corporate social responsibility. En: Harvard Business Review, $\mathrm{n}^{\circ} 84,78-92$.

Sacconi L.; Baldin E. (2003). Come le organizzazioni possono essere socialmente responsabili? En: Sviluppo e organizzazione, $\mathrm{n}^{\circ}$ 196, 49-56.

Sassatelli, R. (2004). Consumo, cultura e società. Bologna: Il Mulino.

Salvini, A.; Cordarz D. (a cura di) (2005). Le trasformazioni del volontariato in Toscana. II Rapporto d'indagine. Firenze: Quaderni CESVOT, nº27.

Salvini, A.; Corchia L. (a cura di) (2012). I bisogni delle organizzazioni di volontariato. Firenze: Cesvot.

Semprini, A. (1993). Marche e mondi possibili. Milano: Angeli.

Solito, L. (2010). Comunico ergo sum. Idee e fatti sulla comunicazione. Firenze: Le Lettere.

Solito, L.; Sorrentino C. (a cura di) (2011). Il volontariato. Immagini, percezioni e stereotipi. Firenze: Ed. Quaderni Cesvot, $n^{0} 50$.

Solito, L.; Materassi L. (2013). Diverse eppur vicine. Firenze: FUP. 
Sorrentino, C. (2008). La società densa. Riflessioni intorno alle nuove forme di sfera pubblica. Firenze: Le Lettere.

Terenzi, P. (2012). Percorsi di sociologia relazionale. Milano: FrancoAngeli.

Thompson, J. B. (1998). Mezzi di comunicazione e modernità. Bologna: Il Mulino.

Zamagni, S. (2004). L'ancoraggio etico della responsabilità sociale d’impresa e la critica alla RSI. Bologna: Dipartimento di Scienze Economiche, Working Paper $\mathrm{n}^{0} 1$.

Zattoni, A.; Perrini F. (2006). Corporate Governance. Milano: Il Sole 24 Ore.

\section{Referencia de este artículo}

Solito, Laura (2014). Relaciones virtuosas. Empresas y asociaciones frente a la Responsabilidad Social Corporativa. En: adComunica. Revista Científica de Estrategias, Tendencias e Innovación en Comunicación, $\mathrm{n}^{\circ} 8$. Castellón: Asociación para el Desarrollo de la Comunicación adComunica, Universidad Complutense de Madrid y Universitat Jaume I, 131-152. DOI: http://dx.doi.org/10.6035/21740992.2014.8.8. 